\title{
Experimental Data Processing Using Shift Methods
}

\author{
Andrey Novikov-Borodin ${ }^{1, \star}$ \\ ${ }^{1}$ Institute for Nuclear Research (INR), Russian Academy of Sciences, Moscow, 117312 Russia
}

\begin{abstract}
The numerical methods of step-by-step and combined shifts are proposed for correction and reconstruction the experimental data convolved with different blur kernels. Methods use a shift technique for the direct deconvolution of experimental data, they are fast and effective for data reconstruction, especially, in the case of discrete measurements. The comparative analysis of proposed methods is presented, inaccuracies of reconstruction with different blur kernels, different volumes of data and noise levels are estimated. There are presented the examples of using the shift methods in processing the statistical data of TOF neutron spectrometers and in planning the proton therapy treatment. The multi-dimensional data processing using shift methods is considered. The examples of renewal the 2D images blurred by uniform motion and distorted with the Gaussian-like blur kernels are presented.
\end{abstract}

\section{Introduction}

A convolution is a widespread transformation in experimental data. It already appears as a result of overlapping the hypothetical ideal momentary signals during the time of the measurements and looks like the signal distortion. A deconvolution is needed to reconstruct the momentary signals from the distorted data to increase the accuracy of the measurements. In respect to mathematics, the deconvolution belongs to the class of ill-posed problems [1,2], and an universal solution does not exist. The possibility of data reconstruction depends on many factors and it makes the development of different reconstruction techniques an actual and challenging research topic. The proposed numerical methods of step-by-step and combined shifts have quite simple algorithms and in a number of cases are more effective for data reconstruction than the existing methods of the direct matrix inversion and the methods based on the integral transformations or the regularization technique $[3,4]$.

\section{Algorithms of shift methods}

When a momentary signal $h(x)$ is convolved with the blur kernel $S(x)$, the measuring system response (without random noise and other distortions) is: $H(x)=(S * h)=\int S(\xi) h(x-\xi) \mathrm{d} \xi=\int S(x-\xi) h(\xi) \mathrm{d} \xi$. In the case of discrete measurements, the functions $S(x)$ and $h(x)$, and the experimental data $H(x)$ may be represented using the Kronecker symbol $\delta_{i}=1$ in the $i$-th interval of the $x$-axis partition as:

$$
h=\sum_{m} h_{m} \delta_{m}, \quad S=\sum_{k} s_{k} \delta_{k}, \quad H=\sum_{p} H_{p} \delta_{p}=\sum_{k, m} s_{k} h_{m} \delta_{k+m} .
$$

^e-mail: novikov.borodin@gmail.com 
The linearity of Eq. (1) with respect to the function $S$ is used in the step-by-step shift method (the $\mathrm{SbS}$ method) for data reconstruction by means of iterative equations:

$$
S^{(n+1)}=S^{(n)}-a_{n} S_{[n+1]}^{(n)}, \quad H^{(n+1)}=H^{(n)}-a_{n} H_{[n+1]}^{(n)}, \quad a_{n}=s_{n+1}^{(n)} / s_{0}, \quad n=0,1, \ldots
$$

Here $H^{(n)}=\sum_{k, m} s_{k}^{(n)} h_{m}^{(n)} \delta_{k+m}$ and $S^{(n)}=\sum_{k} s_{k}^{(n)} \delta_{k}$ are the system response and the blur kernel at the $n$-th iteration step (in the round brackets), $S_{[l]}^{(n)}=\sum_{k} s_{k}^{(n)} \delta_{k+l}$ and $H_{[l]}^{(n)}=\sum_{k, m} s_{k}^{(n)} h_{m}^{(n)} \delta_{k+m+l}$ are the same functions shifted over $l$ discrete intervals (in the square brackets), $S^{(0)}=S, H^{(0)}=H$.

According to the algorithm of the SbS method (2), the first $n$ coefficients in the function $S^{(n)}$ after $n$ iteration steps are consequently put to zero: $s_{i}^{(n)}=0, i=1, \ldots, n$, so inside the interval $[0, n]$ : $S^{(n)}=s_{0} \delta_{0}$ and the data $H^{(n)}=s_{0} \sum_{m} h_{m} \delta_{m}=s_{0} h$ are being reconstructed there.

The SbS method has the similar possibilities of reconstruction as the existing methods of the direct matrix inversion, but one may use the SbS algorithm (2) for reconstruction the response $h(P)$ with respect to the pulse $P$ when the blur kernel is a linear combination of pulses $P=\sum_{i} p_{i} \delta_{i}$ : $S=$ $\sum_{j} s_{j} P_{[j]}$. Here, as before, $P_{[j]}=\sum_{i} p_{i} \delta_{i+j}$ is the pulse $P$ shifted over $j$ intervals.

In particular, if: $S=P+a P_{[l]}$, the iterative equations (2) may be modified as:

$$
S^{(n+1)}=S^{(n)}+a^{2^{n}} S_{\left[2^{n} l\right]}^{(n)}, \quad H^{(n+1)}=H^{(n)}+a^{2^{n}} H_{\left[2^{n} l\right]}^{(n)},
$$

where $n=1,2, \ldots, S^{(1)}=S^{(0)}-a S_{[l]}^{(0)}, H^{(1)}=H^{(0)}-a H_{[l]}^{(0)}, S^{(0)}=S, H^{(0)}=H$. Only $N^{*}=\log _{2}(N / l)$ iteration steps by the modified method (3) are needed to reconstruct the data on the interval $[0, N]$ instead of $N$ steps needed by the SbS method (2).

The modified method (3) was used to reconstruct the TOF neutron spectrometer experimental data in the set 2 of measurements with respect to the first pulse (see Fig. 1A). Here $S=P+a P_{[l]}, a \approx 0.5$, $l \approx 50$, and only three iterations were needed to reconstruct the data inside the considered interval of length $N=400$. As far as the coefficient $a=0.5<1$, the term $a^{2^{n}} H_{\left[2^{n} l\right]}^{(n)}$ in Eq. (3) rapidly decreases with iteration steps. After three iterations: $a^{2^{3}}=0.004$, so the accuracy of data reconstruction in other intervals is $\sim 0.4 \%$ (see $[5,6]$ for details).
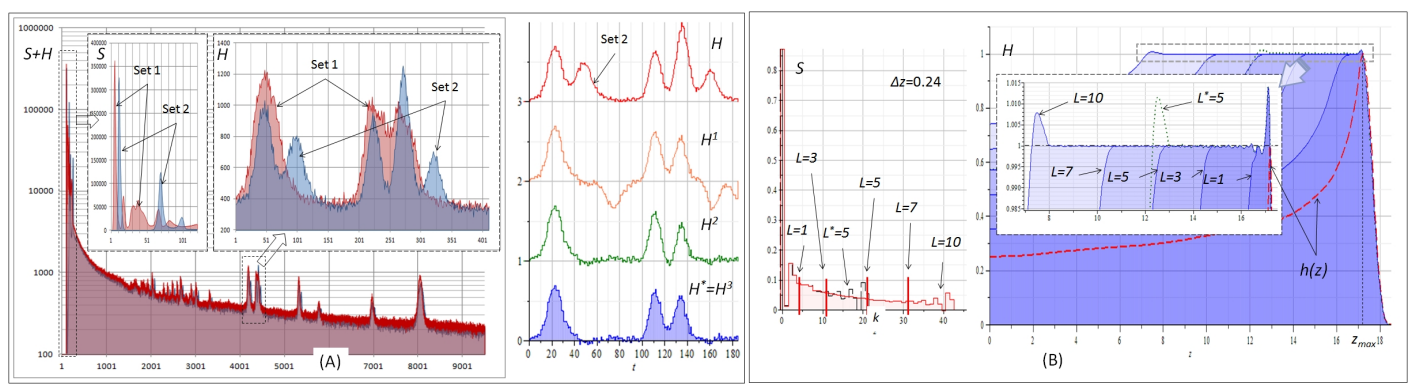

Figure 1. The reconstruction of TOF neutron spectrometer experimental data (A) and the dose distribution modeling at Proton therapy complex in INR RAS

By default, the SbS method reconstructs the data with respect to the element $s_{0}$ of the blur kernel $S$, and if $s_{0}$ is not a maximal element, the coefficients in Eqs. (2), (3) $a_{n}, a>1$ and the term $a^{2^{n}} H_{\left[2^{n} l\right]}^{(n)}$ in Eq. (3) increases exponentially with iteration steps. For $a=1.01$ after 25 iterations: $a^{25}=1.75$. $10^{145001}$ and after about 20-30 iterations the numerical calculations will be interrupted because of the machine number maximal value overflow. The random noise factor in experimental data worsens considerably this situation (see [6] for details). 
It is proposed to solve this problem with the help of the method of combined shifts (the CS method). According to the CS algorithm, the linear combination of the shifted functions $S_{[i]}=$ $\sum_{k=0}^{K} s_{k} \delta_{k+i}$ with some coefficients $\alpha_{i}$ is used to get a new kernel $S^{*}=\sum_{i=-L}^{L} \alpha_{i} S_{[i]}$, in which $L$ coefficients around the maximal term $s_{k_{m}}^{*}=s_{k_{m}}$ are put to zero. If $M<\infty$ in $h=\sum_{m=0}^{M} h_{m} \delta_{m}$ and when $L>M$, the response from the $k_{m}-L-1$-th term of $S^{*}$ cannot overlap the response from the $k_{m}$ term, so in the interval $\left[k_{m}, k_{m}+L\right]: H^{*}=\sum_{i=-L}^{L} \alpha_{i} H_{[i]}=s_{k_{m}} h$ and the data are reconstructed with respect to the maximal element of the kernel $S$.

The unknown coefficients $\boldsymbol{\alpha}=\left\{\alpha_{i}\right\}_{i=-L}^{L}$ may be found as the solutions of a linear system of equations: $\boldsymbol{\alpha}=\mathbf{S}^{*} \cdot \mathbf{M S}^{-1}$, where $\mathbf{M S}^{-1}$ is the inverse to the shift matrix $\mathbf{M S}=\left\{s_{k_{m}+i-j}\right\}_{i, j=-L}^{L}$ and the vector $\mathbf{S}^{*}=\left\{s_{i}^{*}\right\}_{i=-L}^{L}$ corresponding to $S^{*}$ has only one non-zero element $s_{0}^{*}=s_{k_{m}}$. Thus, the method of combined shifts is built with the help of the equations:

$$
H^{*}=\sum_{i=-L}^{L} \alpha_{i} H_{[i]}, \quad \alpha=\mathbf{S}^{*} \cdot \mathbf{M S}^{-1} .
$$

The data are reconstructed with respect to the maximal term $s_{k_{m}}=s_{\max }$ of the kernel $S$, so the CS method (4) may be used in cases, where solutions of the SbS method and the methods of the direct matrix inversion diverge. For this reason the CS method is also slightly sensitive to the random noise in the data $H$. Usually, the noise levels in the initial $H$ and reconstructed $h$ data are of the same order (see examples in [6]). The CS method differs from the direct matrix inversion methods and is a good alternative to the regularization technique in solving the ill-posed deconvolution problem.

The SbS and CS methods may also be used for processing the continuous functions. An example of using the combined shift method for modeling the dose distribution $H(z)$ with the flat shelves of length $L=1,3,5,7,10 \mathrm{~cm}$ needed for the proton therapy treatment [7] is shown in Fig. 1B. The dose distribution $H(z)$ is a linear combination of Bragg curves $h(z)$ shifted by $k \Delta z$ steps with coefficients $\mathbf{S}=\left\{s_{k}\right\}_{k=0}^{N}$, which were found according to Eq. (4) as a solution of the linear system of equations: $\mathbf{S}=\mathbf{H} \cdot \mathbf{M H}^{-1}$, where the distribution $\mathbf{H}=\left\{H\left(z_{n}\right)\right\}_{n=0}^{N}$ is specified in given points $z_{n}, \mathbf{M H}^{-1}$ is the inverse to the matrix $\mathbf{M H}=\left\{h\left(z_{\max }+(n-k) \Delta z\right)\right\}_{k, n=0}^{N}$, where $z_{\max }$ is the coordinate of the Bragg peak.

\section{Image renewal}

The proposed $1 D$ shift methods may also be used for reconstruction the multi-dimensional data or images:

$$
H(\boldsymbol{x})=(S * I)=\int \cdots \int S(\boldsymbol{\xi}) I(\boldsymbol{x}-\boldsymbol{\xi}) \mathrm{d} \boldsymbol{\xi}
$$

distorted with the kernel $S(\boldsymbol{x})$, where $\boldsymbol{x}=\left\{x_{i}\right\}_{i=1}^{n}, \boldsymbol{\xi}=\left\{\xi_{i}\right\}_{i=1}^{n}$ and $\mathrm{d} \boldsymbol{\xi}=\mathrm{d} \xi_{1} \mathrm{~d} \xi_{2} \cdots \mathrm{d} \xi_{n}$, if the multiple integrals in $H(\boldsymbol{x})$ may be represented as a combination of $1 D$ integrals (see [6] for details).

For example, when an image $H(\boldsymbol{x})$ or objects in it are smeared along with some direction (say, along with the $x_{i}$-axis), Eq.(5) may be represented as a $1 D$-integral: $H(x)=\left(S\left(x_{i}\right) * I\right)=$ $\int S\left(\xi_{i}\right) I\left(\ldots, x_{i}-\xi_{i}, \ldots\right) \mathrm{d} \xi_{i}$, so the $1 D$ shift methods may be used for the image renewal.

In the important particular case, when the objects in the image $H=H^{0}$ are smeared by the uniform motion over $l=20$ pixels with kernel $S=\sum_{i=0}^{19} \delta_{i}$ over the $x$-axis (see Fig. 2A), only four iterations are needed to reconstruct the image with $N=100$ pixels width. Indeed, after the first iteration: $S^{(1)}=S-S_{[1]}=\delta_{0}-\delta_{20}$, and only $3\left(\geq \log _{2}(100 / 20)\right)$ additional steps are needed to renew the image using the modified method (3). The reconstruction accuracy ( $\max \left|H^{*}-I\right|$ ) is $\sim 5 \cdot 10^{-15}$.

The multiple integral (5) may be represented as a combination of $1 D$ integrals: $I_{1}, I_{2}, \ldots, I_{n}=I$, when the blur kernel may be factored: $S(\boldsymbol{x})=S_{1}\left(x_{1}\right) S_{2}\left(x_{2}\right) \cdots S_{n}\left(x_{n}\right)$ :

$$
H(\boldsymbol{x})=\left(S_{1} * I_{1}\right), \quad I_{1}(\boldsymbol{x})=\left(S_{2} * I_{2}\right), \ldots \quad I_{n-1}(\boldsymbol{x})=\left(S_{n} * I\right) .
$$




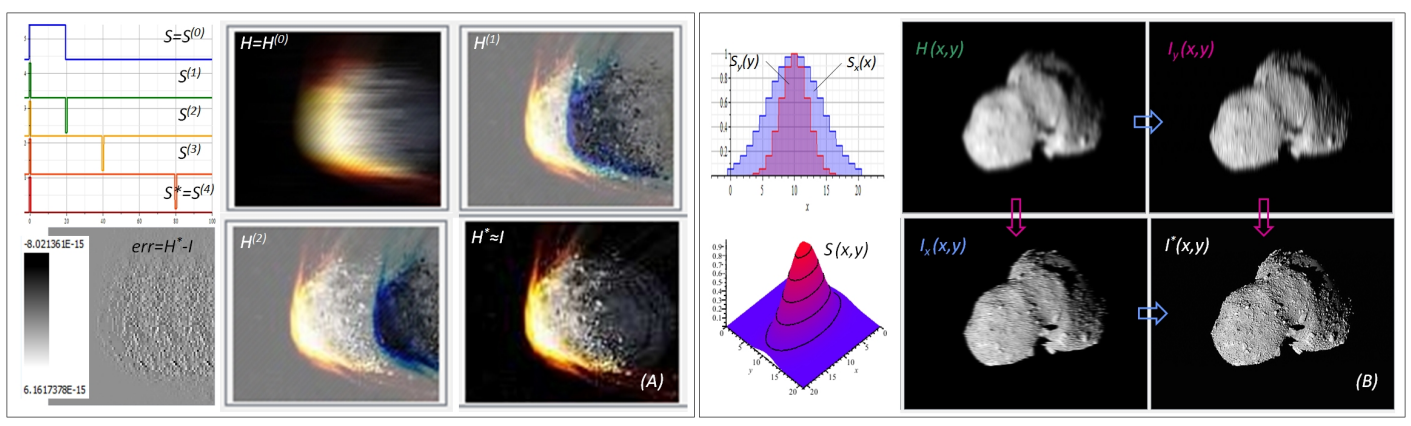

Figure 2. Image renewal using shift methods: (A) smeared by uniform motion, (B) distorted with a Gaussian-like blur kernel

One can renew $H$ consequently reconstructing the images $I_{1}, I_{2}, \ldots, I$. An example of using the CS method (4) for renewing a $2 D$ image distorted with the Gaussian-like blur kernel $S(x, y)=$ $\exp \left(-x^{2} / 2 \sigma_{x}^{2}-y^{2} / 2 \sigma_{y}^{2}\right)$ is shown in Fig. $2 \mathrm{~B}$. The reconstruction error $\left(\max \left|I^{*}-I\right|\right)$ is about $1.5 \%$.

\section{Conclusion}

The numerical methods of step-by-step and combined shifts are proposed for the discrete data processing by means of a shift technique. They are fast, simple to implement and do not require significant computational resources. In many cases frequently met in practice, especially for discrete measurements, the proposed methods are much faster, more accurate and effective than existing numerical methods of the direct matrix inversion and the methods using the regularization technique or based on the integral transformations.

The proposed one-dimensional shift methods may be effectively used for the multi-dimensional data reconstruction and the image renewal. The important for practice examples of renewing the $2 D$ images smeared by uniform motion and distorted with the Gaussian-like blur kernels were considered. The proposed shift methods may be interesting in mathematics as an effective technique for solving the deconvolution problem in the case of discrete and discontinuous functions.

\section{References}

[1] Digital Signal Processing. Handbook / ed. V.K. Madisetti and D.B. Williams (Chapmann \& Hall, 1999)

[2] A.N. Tikhonov, V.Y. Arsenin, Solutions of Ill-posed Problems (Winstons \& Sons, Washington, D.C., 1977)

[3] S.W. Smith, The Scientist and Engineer's Guide to Digital signal processing / 2nd ed. (California Technical Publishing, San Diego, CA, 1999)

[4] R. Gonzalez, Digital Image Processing / 3rd ed. (Pearson Hall., 2008)

[5] A.V. Novikov-Borodin, Instruments and Experimental Techniques 61, 6, 780-787 (2018)

[6] A.V. Novikov-Borodin, Instruments and Experimental Techniques 62, 4, 480-488 (2019)

[7] E. Pedroni, S. Scheib, T. Bohringer, A. Coray, M. Grossmann, S. Lin, A. Lomax, Physics in Medicine and Biology 50, 541-561 (2005) 